\title{
Eine inhaltsreiche Grammatik des Udmurtischen
}

EBERHARD WINKLER: Udmurtische Grammatik. Veröffentlichungen der Societas Uralo-Altaica, Band 81. Wiesbaden: Harrassowitz Verlag, 2011. $188 \mathrm{~S}$.

Für die finnisch-ugrischen Sprachen Russlands liegen nach wie vor verhältnismäßig wenig gute Grammatiken in Weltsprachen - oder überhaupt in irgendeiner Sprache - vor, obwohl in den letzten Jahrzehnten vor allem in Finnland und Ungarn einige Lehrbücher erstellt wurden. So füllt die neue udmurtische Grammatik von Eberhard Winkler, Professor für Finnougristik an der Universität Göttingen, eine erhebliche Lücke. Winkler erstellte 2001 für die Europa-Reihe von Lincom (Languages of the World/Materials 212) den grammatischen Abriss Udmurt, der weniger als 100 Seiten umfasste. Auch dieser
Abriss war brauchbar, aber knapp und stellenweise zu sehr an die einheitliche Instruktion der Reihe gebunden; im Vergleich zu dem nun vorliegenden Band handelt es sich um ein Skelett.

Die Udmurtische Grammatik gliedert sich in vier Hauptkapitel: Phonologie, Morphonologie, Morphologie und Morphosyntax, Syntax. In Stil und Darstellungsweise ist die Grammatik äußerst komprimiert: Die Phänomene werden exakt und kurz beschrieben, und der Text weist keinerlei Redundanz auf. Zahlreiche Querverweise zwischen den Kapiteln erleichtern das Auffinden bereits gegebener oder an späterer Stelle folgender Informationen, was Wiederholungen überflüssig macht. Der Sachinhalt ist übersichtlich in Kapitel und zahlreiche Unterkapitel gegliedert. Diese Kompaktheit macht die Gram- 
matik benutzerfreundlich, denn die gesuchten Punkte sind dank der Gliederung schnell zu finden. Der Text wird durch zusammenfassende Tabellen u. a. über die Deklination ergänzt.

In der Einleitung beschreibt Winkler u. a. die heutige Lage des Udmurtischen, Dialekte und Sprachkontakte. Er erklärt, das Beispielmaterial der Grammatik stamme aus in Untersuchungen, Grammatiken und Lehrbüchern veröffentlichten Texten und von Informanten. Leider finden sich keine Anmerkungen mit Hinweisen darauf, welche Beispiele von Informanten und welche aus der Literatur stammen. Zum Teil fehlen sie wohl aus Platzgründen, denn jeder Beispielsatz nimmt viele Zeilen in Anspruch: Zuerst wird das udmurtische Beispiel in phonetischer Transkription gegeben, dann darunter dasselbe in Petit mit kyrillischen Buchstaben, danach folgt die Glossierung und als viertes die Übersetzung. An sich ist zu begrüßen, dass auch die kyrillische schriftsprachliche Form wiedergegeben wird. Die Glossierung ist heute auch in der Finnougristik bereits ein must, obwohl sie in einer so stark agglutinierenden Sprache bisweilen anstrengend ist. Winkler verwendet eine rationale Glossierungsweise und verzichtet beispielsweise darauf, Konjunktionen (z. B. ma-li ke šu-ono [was-DAT if say-PART] 'weil') in ihre morphologischen Teile zu zerlegen.

Im Phonologie-Kapitel beschreibt Winkler neben der Qualität der Laute auch ihre Frequenz aufgrund der vorliegenden Untersuchungen. Obwohl die Grammatik auf der Schriftsprache basiert, gibt er in kleingedruckten Abschnitten auch Informationen über die Repräsentation in den Dialekten. Bei den Vokalen hätte die Beschreibung der sehr unterschiedlichen PhonemInventarien der Dialekte unmittelbar nach Punkt 1 des Vokal-Kapitels (Inventar) einen sinnvolleren Platz gefunden (wie beim Konsonantismus) als am Ende des gesamten Kapitels. Die Laute werden in der Regel in der finnisch-ugrischen Transkription dargestellt, aber in den Tabellen werden auch die IPAEntsprechungen angegeben. Das Buch weist nur wenige Druckfehler auf, doch leider finden sich einige gerade in der Darstellung des Konsonantismus: In der Tabelle wurde das Zeichen für die stimmhafte palatale Affrikate mit dem Zeichen für den entsprechenden stimmlosen Laut vertauscht. In einem Teil der Anmerkungen auf der folgenden Seite wird anstelle der finnischugrischen Transkription die IPA verwendet. Stellenweise wird später im Text das $\chi$ nach deutscher Art mit ch markiert. 
Im Morphonologie-Kapitel werden alle in der Deklination auftretenden Lautwechsel sowohl im Konsonantismus wie im Vokalismus genauestens beschrieben. Viele Verfasser von Grammatiken beschränken sich auf eine rein synchrone Darstellung. Winkler hat sich glücklicherweise für einen Kompromiss entschieden: In Fällen, in denen es schwierig wäre, eine bestimmte Eigenschaft oder Form ohne die Kenntnis ihrer Geschichte $\mathrm{zu}$ verstehen, gibt er in aller Kürze - in einem Nebensatz oder in Klammern - eine diachrone Erklärung. Stellenweise verweist er auch auf die Struktur anderer uralischer Sprachen. Diese Lösung liegt unbedingt im Interesse des Lesers.

Das Kapitel Morphologie und Morphosyntax beginnt traditionsgemäß mit den Substantiven und schreitet über die anderen Nominalklassen zu den Verben fort. Dieses Kapitel nimmt natürlich den größten Teil des Bandes, mehr als hundert Seiten, in Anspruch und befasst sich auch mit der Wortbildung. Vor der eigentlichen Deklination stellt Winkler die Eigenschaften und die Verwendung der Wortstämme und Suffixe im Allgemeinen dar. Das in der Suffixhäufung auftretende Possessivsuffix der 3. Person Singular analysiert er als Nominalisierer, der aus einem Wort gewissermaßen eine neue, wie ein Substantiv fungierende Form macht, an die wiederum Kasusendungen angefügt werden können.

Die Kasusendungen mit ihren Varianten werden in einer Tabelle dargestellt und in den darauf folgenden Anmerkungen erläutert. Winkler zitiert die neuesten Untersuchungen, u. a. Svetlana Edygarovas Dissertation über die Kategorie der Possessivität im Udmurtischen (2010) und Natal'ja Kondrat'evas Doktorarbeit über die Kasuskategorien des Udmurtischen (2011). (Beide werden in dieser Ausgabe der FUF besprochen.) Neben der Diachronie erwähnt Winkler die Lehnherkunft einzelner Phänomene, dies jedoch nicht systematisch. Beispielsweise dürfte es sich bei der Verbindung des Ausdrucks 'Udmurtisch lehren/lernen' mit dem Dativ um eine Lehnübersetzung der Rektion der entsprechenden russischen Verben (учить/ся чему) handeln.

Winkler ist bestrebt, die Funktionen der grammatischen Elemente so genau zu beschreiben wie dies auf der Basis der bisherigen Forschung möglich ist; beispielsweise gliedert er die Funktionen der Possessivsuffixe in sechs Gruppen: Possessor, Kongruenzträger, Referenzträger, Vokativ, „Definitheit“, Determinierung, und erwähnt darüber hinaus zwei nur aus der Geschichte der Suffixe zu verstehende 
Aufgaben, nämlich Akkusativ und Nominalisierung. Bei den Pronomina weist er auf die Teilung in ein exklusives 'wir'-Pronomen $(m i)$ und seine inklusive Entsprechung (aśmeos) hin. Generell enthält die vorliegende Grammatik eine ungeheure Menge detaillierter Informationen über verschiedene Formen und ihre Verwendung, obwohl Winkler häufig feststellen muss, dass der Forschungsstand für endgültige Schlussfolgerungen unzureichend ist.

Die nominale Derivation im Udmurtischen hält Winkler für schwach entwickelt und weist darauf hin, dass dies auch in anderen fiu. Sprachen keine Seltenheit ist. Dasselbe kann man ja zum Beispiel für die mordwinische Sprache feststellen, nicht aber für das Mari, das wiederum in vielen anderen Bereichen dem Udmurtischen gleicht.

Anders als in der LincomGrammatik stellt Winkler alle vier synthetischen und fünf analytischen Tempusformen des Udmurtischen als ein System dar, wenngleich er erwähnt, dass einige dieser Formen eine evidentiale Bedeutung einschließen. Er behandelt den Ausdruck der Evidentialität im Udmurtischen separat und teilt ihn in drei Gruppen: Evidential, Inferential, Mirativ. Im Udmurtischen werden gleichartige Verbgefüge verwendet wie im Mari; in beiden Sprachen gehen sie auf türkischen Einfluss zurück. Im Mari ist die Anzahl der finiten modifizierenden Verben dieser Konstruktion hoch, bis $\mathrm{zu} 40$, während im Udmurtischen das System schwächer entwickelt ist und einigen Forschern zufolge nur 7, nach Ansicht anderer weniger als 30 Verben in Gebrauch sind. In beiden Sprachen bringen sie die Aktionsart zum Ausdruck, im Mari auch den Aspekt. Im Rahmen der verbalen Derivation betrachtet Winkler neben der Aktionsart u. a. Simulative ('so tun als ob'), Integration von entlehnten Verben, Objektinkorporation, Empfindungsverbgefüge und Funktionsverbgefüge, denen in Grammatiken häufig keinerlei Beachtung geschenkt wird. Auch die übrigen Wortarten, d. h. alles von den Adverbien an, werden recht detailliert betrachtet und nicht als nebensächlich behandelt wie in vielen anderen Grammatiken.

Die Syntax gliedert sich in vier Hauptgruppen: Satzarten, Satzmodi, der einfache Satz und der zusammengesetzte Satz. In der ersten Gruppe stellt Winkler verschiedene Nominalsatztypen und modale Konstruktionen genauer vor. Bei den Satzmodi werden fragende, befehlende u. ä. Satztypen betrachtet. $\mathrm{Zu}$ den Beispielen für Befehlssätze stellt Winkler fest, dass das Subjekt, das in einigen von ihnen auftritt, nicht gewichtet ist, sondern ver- 
wendet wird, wenn der Satz auch ein Objekt enthält. Als Beweis für diese Behauptung sind die Beispielsätze wenig geeignet. Der Abschnitt Der einfache Satz gibt neben der Beschreibung aller Satzglieder Auskunft über die Wortfolge. Bei der Beschreibung des Objekts werden Verben erwähnt, die immer ein definites Objekt fordern, wie bićatini 'kitzeln, jucken (tr.)', z. B. pidze bićate 'das Bein juckt' (wörtl.: '(etwas) juckt sein Bein'). Meist wird in diesen Fällen die Definitheit wohl mit dem Possessivsuffix angezeigt. Handelt es sich um eine Rektion des Verbs oder um einen Satztyp, nämlich um eine in den fiu. Sprachen typische, physische und psychische Zustände ausdrückende Konstruktion, in der das Subjekt fehlt, obwohl der Satz ein transitives Verb und zudem ein Objekt enthält?

Bei der Darstellung der zusammengesetzten Sätze werden neben den eigentlichen Nebensätzen und den infiniten Konstruktionen die aus Nomen actionis und Postposition gebildeten Konstruktionen berücksichtigt, die von ihrer Funktion her zweifellos den Satzäquivalen- ten nahestehen. Separat behandelt Winkler die Objektsätze der Verba dicendi, unter denen sich - wie im Mari - alles zwischen direkter und indirekter Rede findet. In beiden Sprachen hat sich, offenbar nach dem Vorbild des Tatarischen, das Gerundium des Verbs 'sagen' zur Konjunktion 'dass' entwickelt.

Die Udmurtische Grammatik ist eine hervorragende wissenschaftliche Grammatik, klar aufgebaut und handlich. Winkler hat in seinem Werk nahezu das gesamte Wissen komprimiert, das über die verschiedenen Ebenen der udmurtischen Sprache heute verfügbar ist, und zudem die Punkte aufgezeigt, an denen eine genaue Beschreibung weitere Untersuchungen erfordert. Als zusätzlichen Bonus gibt er Informationen über die Geschichte der Sprache, sofern sie für das Verständnis eines sprachlichen Phänomens unentbehrlich sind. Die Grammatik wird von Studenten und Forschern des Udmurtischen sowie zweifellos auch von Typologen intensiv genutzt werden.

Sirkka Saarinen 